ARTICLE

https://doi.org/10.1038/s41467-019-11914-2

\title{
Topological magnon amplification
}

\author{
Daniel Malz (1) ${ }^{1}$, Johannes Knolle ${ }^{2} \&$ Andreas Nunnenkamp (iD ${ }^{3}$
}

Topology is quickly becoming a cornerstone in our understanding of electronic systems. Like their electronic counterparts, bosonic systems can exhibit a topological band structure, but in real materials it is difficult to ascertain their topological nature, as their ground state is a simple condensate or the vacuum, and one has to rely instead on excited states, for example a characteristic thermal Hall response. Here we propose driving a topological magnon insulator with an electromagnetic field and show that this causes edge mode instabilities and a large non-equilibrium steady-state magnon edge current. Building on this, we discuss several experimental signatures that unambiguously establish the presence of topological magnon edge modes. Furthermore, our amplification mechanism can be employed to power a topological travelling-wave magnon amplifier and topological magnon laser, with applications in magnon spintronics. This work thus represents a step toward functional topological magnetic materials.

\footnotetext{
${ }^{1}$ Max-Planck-Institut für Quantenoptik, Hans-Kopfermann-Strasse 1, D-85748 Garching, Germany. ${ }^{2}$ Blackett Laboratory, Imperial College London, London SW7 2AZ, UK. ${ }^{3}$ Cavendish Laboratory, University of Cambridge, Cambridge CB3 OHE, UK. Correspondence and requests for materials should be addressed to D.M. (email: daniel.malz@mpq.mpg.de)
} 
W hile fermionic topological insulators have a number of clear experimental signatures accessible through linear transport measurements $s^{1,2}$, noninteracting bosonic systems with topological band structure have a simple condensate or the vacuum as their ground state ${ }^{3}$, making it more difficult to ascertain their topological nature. Their excited states, however, may carry signatures of the topology of the band structure, for example, in form of a thermal Hall response ${ }^{4-6}$. There is great interest in certifying and exploiting topological edge modes in bosonic systems, as they are chiral and robust against disorder, making them a great resource to realize backscattering-free waveguides ${ }^{7,8}$ and potentially topologically protected travellingwave amplifiers 9 . It has been predicted that topological magnon insulators (TMI) are realized, e.g., in kagome planes of certain pyrochlore magnetic insulators as a result of DzyaloshinskiiMoriya (DM) interaction ${ }^{4,10,11}$. To date, there exists only indirect experimental proof, via neutron scattering measurements of the bulk band structure in $\mathrm{Cu}[1,3 \text {-benzenedicarboxylate }(\mathrm{bdc})]^{12}$, and observation of a thermal magnon Hall effect in $\mathrm{Lu}_{2} \mathrm{~V}_{2} \mathrm{O}_{7}{ }^{5}$ and $\mathrm{Cu}$ $(1,3-b d c)^{6}$. The main obstacle is that magnons are uncharged excitations and thus invisible to experimental tools like STM or ARPES with spatial resolution. An unambiguous experimental signature, such as the direct observation of an edge mode in the bulk gap is hampered by limitations in energy resolution (in resonant X-ray scattering) or signal strength (in neutron scattering) $)^{12}$.

Here, we propose driving a magnon edge mode to a parametric instability, which, when taking into account nonlinear damping, induces a non-equilibrium steady state with a large chiral edge mode population. In such a state the local polarization and magnetization associated with the edge mode are coherently enhanced, which could enable direct detection of edge modes via neutron scattering. Crucially, we show that selective amplification of edge modes can be achieved while preserving the stability of the bulk modes and thus the magnetic order. Another key experimental signature we predict is that applying a driving field gradient gives rise to a temperature gradient along the transverse direction, thus establishing what one might call a driven Hall effect (DHE). Topological magnon amplification has further uses in magnon spintronics ${ }^{13}$, providing a way to amplify magnons and to build a topological magnon laser ${ }^{14,15}$. Our work on driving topological edge modes in magnetic materials complements previous investigations in ultracold gases ${ }^{16,17}$, photonic crystals ${ }^{9}$, and most recently arrays of semiconductor microresonators ${ }^{14,15}$ and graphene ${ }^{18}$.

\section{Results}

Edge mode parametric instability. Before discussing a microscopic model, we show qualitatively how a parametric instability may arise from anomalous magnon pairing terms in a chiral onedimensional waveguide. We consider bosonic modes $\left\{\hat{a}_{k}\right\}$ with energies $\left\{\omega_{k}\right\}$ (for example the magnon edge mode between the first and second band, as in Fig. 1), labelled by momentum $k$, interacting with another bosonic mode (electromagnetic field mode) $\hat{b}$, as described by generic three-wave mixing $(\hbar=1)$

$$
\hat{H}_{\text {int }}=\sum_{k} \frac{g_{k}}{2}\left(\hat{a}_{-k}^{\dagger} \hat{a}_{k}^{\dagger} \hat{b}+\hat{b}^{\dagger} \hat{a}_{k} \hat{a}_{-k}\right) \text {. }
$$

Under strong coherent driving, the bosonic annihilation operator $\hat{b}$ can be replaced by its classical amplitude $\hat{b} \approx \beta \exp \left(-i \Omega_{0} t\right) \gg 1$, yielding an effective Hamiltonian $\hat{H}=\hat{H}_{0}+g_{k} \beta\left[\hat{a}_{-k}^{\dagger} \hat{a}_{k}^{\dagger} \exp \left(i \Omega_{0} t\right)+\hat{a}_{k} \hat{a}_{-k} \exp \left(-i \Omega_{0} t\right)\right]$. The second term produces magnon pairs with equal and opposite momentum. The time-dependence can be removed by passing to a

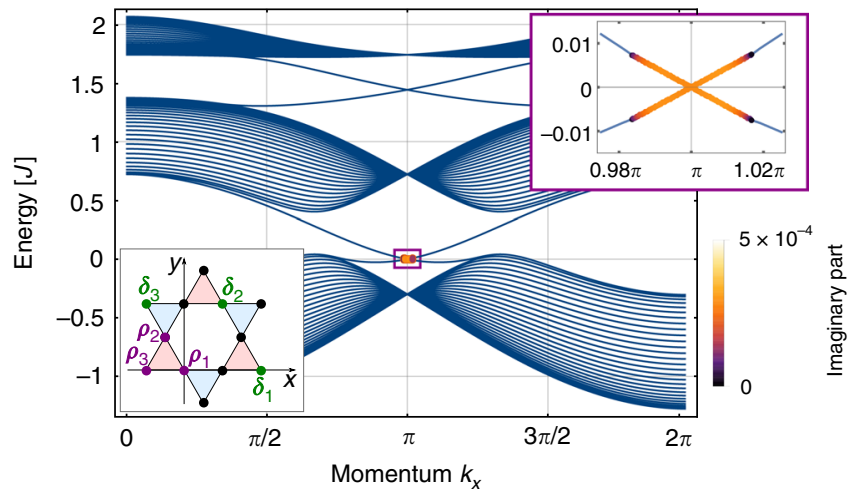

Fig. 1 Magnon band structure and instability. The band structure of a kagome topological magnetic insulator (TMI) strip with a drive tuned to the edge modes at momentum $\pi$ (lattice constant $a=1$ ), calculated from the microscopic model described in the text. Instabilities are marked by coloured disks, with the colour representing the size of the imaginary part, in units of $J$. Bottom left (grey) inset: A sketch of a few unit cells of the kagome lattice, with lattice vectors $\delta_{i}\left(\left|\delta_{i}\right|=a=1\right)$ and site vectors $\rho_{i}$ drawn in. The system we consider is infinite along the $x$-direction and comprises $W=45$ unit cells along the $y$-direction. Top right (purple) inset: Zoom of the unstable region. Parameters: $D_{z} / J=0.2, h / J=0.01, \Omega_{0} / J=2.578$, $\widehat{\mathbf{e}}=(0,1), \mathcal{E} / J=0.0004, \gamma / J=0.0001$

rotating frame with respect to $\sum_{k}\left(\Omega_{0} / 2\right) \hat{a}_{k}^{\dagger} \hat{a}_{k}$. From the Hamiltonian, it is straightforward to derive the equations of motion, which couple particles at momentum $k$ with holes at $-k$. Neglecting fluctuations, we focus on the classical amplitudes of the fields $\alpha_{k}=\left\langle\hat{a}_{k}\right\rangle$ and include a phenomenological linear damping rate $\gamma_{k}$ to take into account the various damping processes present in such materials ${ }^{12,19}$. As we are interested in amplification around a small bandwidth, we neglect the momentum dependence of the coupling $g_{k} \simeq g$ and damping $\gamma_{k} \simeq \gamma$, arriving at

$$
i \frac{d}{d t} \mathbf{A}_{k}=\left(\begin{array}{cc}
\tilde{\omega}_{k}-i \frac{\gamma}{2} & \mathcal{E} \\
-\mathcal{E} & -\tilde{\omega}_{-k}-i \frac{\gamma}{2}
\end{array}\right) \mathbf{A}_{k},
$$

where we have introduced the frequency relative to the rotating frame $\tilde{\omega}_{k}=\omega_{k}-\Omega_{0} / 2$, the vector $\mathbf{A}_{k}=\left(\alpha_{k}, \alpha_{-k}^{*}\right)$, and the overall coupling strength $\mathcal{E}=g \beta$. The eigenvalues of the dynamical matrix Eq. (2) are the complex energies

$$
\omega_{k, \pm}=\frac{\omega_{k}-\omega_{-k}}{2}-\frac{i \gamma}{2} \pm \sqrt{\frac{\left(\tilde{\omega}_{k}+\tilde{\omega}_{-k}\right)^{2}}{4}-\mathcal{E} .}
$$

If the coupling $\mathcal{E}$ exceeds the energy difference between pump photons and magnon pair (the detuning) $\tilde{\omega}_{k}+\tilde{\omega}_{-k}=\omega_{k}+\omega_{-k}-\Omega_{0}$, the square root becomes imaginary. If further its magnitude exceeds $\gamma$, more particles are created than dissipated, causing an instability and exponential growth of the number of particles in this mode. Eventually the growth is limited by nonlinear effects, as discussed below. Despite its simplicity, Eq. (3) provides an accurate account of the fundamental instability mechanism in two-dimensional kagome TMIs, as is illustrated by the quantitative agreement Fig. 2. This forms the key ingredient for directly observing chiral magnon edge modes.

Microscopic model. Turning to a more realistic model, we consider spins on the vertices of an insulating kagome lattice ferromagnet that interact via Heisenberg and Dzyaloshinskii-Moriya 

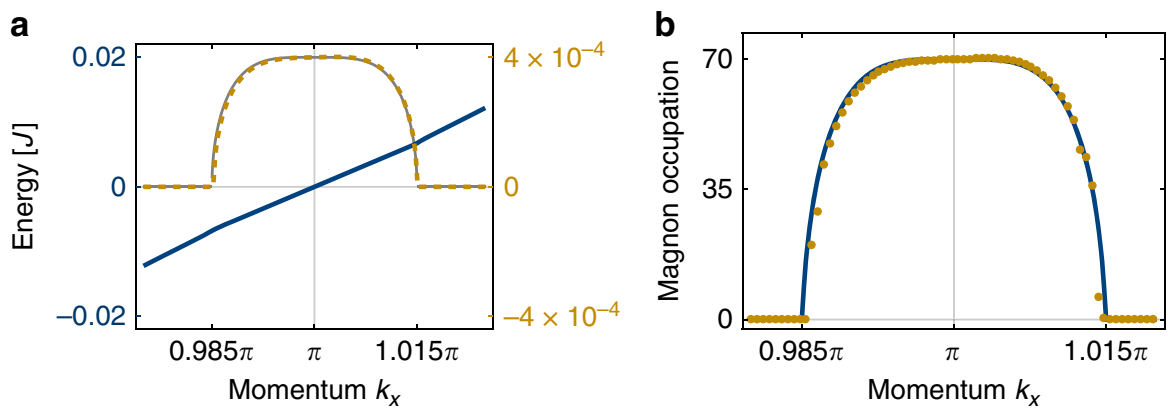

Fig. 2 Characterization of instability. a Complex energy of the edge eigenmodes (cf. Eq. (3)). The real part (frequency) is plotted in blue, whereas the imaginary part (amplification) is shown in yellow. The perfectly matching grey curve is the theory Eq. (3) with $\mathcal{E}=g_{k} E_{0}=\left|\mathbf{E}_{0}\right|\left|\widetilde{\mathbf{Q}}_{\overline{s s}}(\pi)\right|=4 \times 10^{-4} \mathrm{~J}$ and $\omega_{\pi}^{\prime \prime} \simeq 0.3605 \mathrm{~J}$ (numerically extracted from band structure, not fitted to instability). Note that the excellent agreement only holds if the polarization of the electric field points along $y$, i.e., along the width of the strip, as we explain in more detail in Supplementary Note 4. b The steady-state edge occupation calculated from Eq. (8) with the same parameters as in Fig. 1 and nonlinear damping $\eta=10^{-5} \mathrm{~J}$ (the same in microscopic theory and chiral waveguide model). Shown in blue is the chiral waveguide model, the yellow dots are calculated numerically from the microscopic Hamiltonian. Parameters are as in Fig. 1, but with $W=15$

(DM) interaction

$$
\hat{H}_{0}=\sum_{\langle i j\rangle}\left[-J \widehat{\mathbf{S}}_{i} \cdot \widehat{\mathbf{S}}_{j}+\mathbf{D}_{i j} \cdot\left(\widehat{\mathbf{S}}_{i} \times \widehat{\mathbf{S}}_{j}\right)\right]-g_{\mathrm{L}} \mu_{\mathrm{B}} \mathbf{H}_{0} \cdot \sum_{i} \widehat{\mathbf{S}}_{i}
$$

Here, $\mathbf{D}_{i j}$ is the DM vector that can in principle differ from bond to bond, but is heavily constrained by lattice symmetries. $\mathbf{H}_{0}$ is an externally applied magnetic field, $\mu_{\mathrm{B}}$ the Bohr magneton, $g_{\mathrm{L}}$ the Landé g-factor, and $J$ is the Heisenberg interaction strength. This model has been found to describe the thermal magnon Hall effect in $\mathrm{Lu}_{2} \mathrm{~V}_{2} \mathrm{O}_{7}{ }^{5}$, as well as the bulk magnon band structure of $\mathrm{Cu}(1,3-\mathrm{bdc})^{12}$.

The low energy excitations around the ferromagnetic order are magnons, whose bilinear Hamiltonian is obtained from a standard Holstein-Primakoff transformation to order $1 / S$ along the direction of magnetization, i.e., $\hat{S}^{+}=\sqrt{2 s} \hat{a}, \hat{S}^{-}=\sqrt{2 s} \hat{a}^{\dagger}, \hat{S}^{z}=$ $s-\hat{a}^{\dagger} \hat{a}^{4,10}$, yielding

$$
\hat{H}_{0}=-\frac{1}{2}\left(J+i D_{z}\right) \sum_{\langle m n\rangle} \hat{a}_{m}^{\dagger} \hat{a}_{n}+\text { H.c. }+h \sum_{m} \hat{a}_{m}^{\dagger} \hat{a}_{m}+K_{0},
$$

where $K_{0}$ is a constant, the sum ranges over bonds directed counterclockwise in each triangle, and we have chosen the magnetic field to point along $z$, introducing $h \equiv g_{\mathrm{L}} \mu_{\mathrm{B}} H_{0}^{z}$.

To second order, the Hamiltonian only contains the component of $\mathbf{D}_{i j}$ along $z\left(D_{z}\right)$, which is the same for all bonds due to symmetry. We take the unit cell to be one upright triangle (red in Fig. 1), with sites $\rho_{1}=(0,0), \rho_{2}=(-1, \sqrt{3}) / 4, \rho_{3}=(-1 / 2,0)$. The unit cells form a triangular Bravais lattice generated by the lattice vectors $\delta_{1}=(1,0), \delta_{2}=(1, \sqrt{3}) / 2, \delta_{3}=\delta_{2}-\delta_{1}=(-1, \sqrt{3}) / 2$. For nonzero $D_{z}$, the bands in this model are topological ${ }^{10,12}$ causing exponentially localized edge modes to appear within the band gaps.

The effect of an oscillating electric field on magnons in a TMI is characterized by the polarization operator, which can be expanded as a sum of single-spin terms, products of two spins, three spins, etc ${ }^{20}$. Lattice symmetries restrict which terms may appear in the polarization tensor ${ }^{20}$. In the pyrochlore lattice, the polarization due to single spins (linear Stark effect) vanishes, as each lattice site is a centre of inversion, such that the leading term contains two spin operators. The associated tensor can be decomposed into the isotropic (trace) part $\pi$, as well as the anisotropic traceless symmetric and antisymmetric parts $\boldsymbol{\Gamma}$ and $\mathbf{D}$, viz. $\widehat{\mathbf{P}}_{j l}=\left(\pi_{j l} \delta^{\beta \gamma}+\mathbf{\Gamma}_{j l}^{(\beta \gamma)}+\mathbf{D}_{j l}^{[\beta \gamma]}\right) \hat{S}_{j}^{\beta} \hat{S}_{l}^{\gamma}$ (sum over $\beta, \gamma$ implied). Kagome TMIs generically have a nonzero anisotropic symmetric part, which implies the presence of anomalous magnon pairing terms in the spin-wave picture

$$
\begin{aligned}
\mathbf{P}_{m n} & =\left(\boldsymbol{\Gamma}_{m n}^{x x}-\Gamma_{m n}^{y y}-2 i \Gamma_{m n}^{(x y)}\right) \hat{a}_{m} \hat{a}_{n}+\cdots \\
& \equiv \mathbf{Q}_{m n} \hat{a}_{m} \hat{a}_{n}+\text { H.c. }+\cdots .
\end{aligned}
$$

The polarization enters the Hamiltonian via coupling to the amplitude of the electric field, $\hat{H}(t)=\hat{H}_{0}-\mathbf{E}(t) \cdot \widehat{\mathbf{P}}$, thus introducing terms that create a pair of magnons while absorbing a photon. Pair production of magnons is a generic feature of antiferromagnets (via $\pi)^{20}$, but since in ferromagnets it relies on anisotropy, it is expected to be considerably weaker. A microscopic calculation based on a third-order hopping process in the Fermi-Hubbard model at half filling reveals that $|\mathbf{Q}|=a e$ $(t / U)^{3} / 2$, where $a$ is the lattice vector, $e$ the elementary charge, $t$ the hopping amplitude, and $U$ the on-site repulsion (see Supplementary Note 1).

As in the chiral waveguide model, assume an oscillating electric field $\mathbf{E}(t)=\mathbf{E}_{0} \cos \left(\Omega_{0} t\right)$. We consider an infinite strip with $W$ unit cells along $y$, but remove the lowest row of sites to obtain a manifestly inversion-symmetric model. Note that this is a choice out of convenience and that inversion symmetry is by no means a requirement for our scheme. Diagonalizing the undriven Hamiltonian $\hat{H}_{0}(4)$, we label the eigenstates $b_{k, s}$ by their momentum along $x$ and an index $s \in\{1,2, \cdots, 3 W-2\}$. After performing the rotating-wave approximation, the full Hamiltonian reads

$$
\hat{H}=\sum_{k, s} \tilde{\omega}_{k, s} \hat{b}_{k, s}^{\dagger} \hat{b}_{k, s}-\frac{1}{2}\left[\mathbf{E}_{0} \cdot \widetilde{\mathbf{Q}}_{s s^{\prime}}(k) \hat{b}_{k, s} \hat{b}_{-k, s^{\prime}}+\text { H.c. }\right]
$$

where we have introduced $\tilde{\omega}_{k, s}=\omega_{k, s}-\Omega_{0} / 2$, and $\widetilde{\mathbf{Q}}_{s s^{\prime}}(k)$, which characterizes the strength of the anomalous coupling between two modes. It is obtained from $\mathbf{Q}_{m n}$ through Fourier transform and rotation into the energy eigenbasis (cf. Supplementary Note 3 ).

As in the one-dimensional waveguide model, a pair of modes is rendered unstable if their detuning $\Delta_{k, s s^{\prime}}=\omega_{k, s}+\omega_{-k, s^{\prime}}-\Omega_{0}$ is smaller than the anomalous coupling between them. The detuning $\Delta_{k, s s^{\prime}}$ varies quickly as a function of $k$ except at points where the slopes of $\omega_{k, s}$ and $\omega_{-k, s^{\prime}}$ coincide to first order, which happens at $k=0, \pi$ when $s=s^{\prime}$. At those values of $k$, the energy matching condition is fulfilled for a broader range of wavevectors, which leads to a larger amplification bandwidth. However, the edge modes are only localized to the edge around $k=\pi$, such that driving around $k=\pi$ is most efficient, which we consider here ( $c f$. 
a

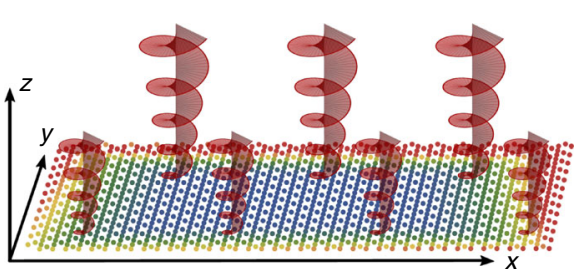

Net energy current along edges b

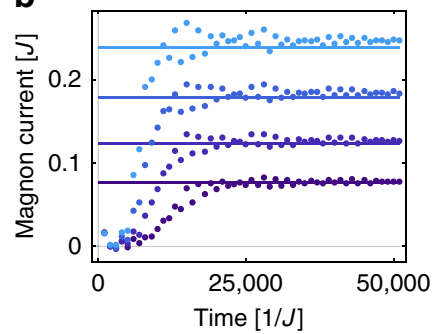

C

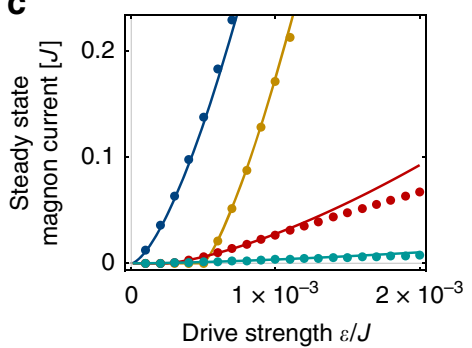

Fig. 3 Driving and resulting edge current. a The two-dimensional kagome lattice ferromagnet is driven by an electromagnetic field perpendicular to the kagome plane. To observe the driven Hall effect (DHE), the field is applied with a linear gradient along $y$, which leads to a temperature difference along $x$. Colour gradient from blue to red indicates increasing temperature. $\mathbf{b}$ The current as function of time. The shades of blue from dark to bright correspond to $\mathcal{E}=\{3,4,5,6\} \times 10^{-4} \mathrm{~J}$, respectively. The straight line is the theoretical prediction for the steady-state value (10). From the equation of motion one can estimate the time to reach the steady state to be of order $t_{\mathrm{eq}} \sim \mathcal{E}^{-1} \log (\mathcal{E} / \eta)$. c The steady-state particle current plotted against the drive strength $\mathcal{E}$. The solid lines correspond to the theoretical formula Eq. (10), whereas the dots are calculated numerically. In $\{$ blue, yellow, red, turquoise $\}$ we show $(\gamma, \eta)=\{(1$, $0.1),(100,0.1),(50,1),(10,10)\} \times 10^{-5} \mathrm{~J}$. We see that Eq. (10) agrees well with the numerically calculated steady-state current. The yellow, red, and turquoise curves have kinks at $2 \mathcal{E}=\gamma$, which mark the onset of instability. The clear deviation occurs once bulk modes become unstable, in which case our approximations break down and system ceases to remain close to the ground state. All unspecified parameters are as in Fig 1. , except for $W=15$

Fig. 1). Expanding the dispersion to second order around this point, we find $\omega_{\pi+q} \simeq \omega_{\pi}+q \omega_{\pi}^{\prime}+\left(q^{2} / 2\right) \omega_{\pi}^{\prime \prime}+\mathcal{O}\left(q^{3}\right)$, yielding $\Delta_{\pi+q}=2 \omega_{\pi}-\Omega_{0}+q^{2} \omega_{\pi}^{\prime \prime}+\mathcal{O}\left(q^{4}\right)$. Placing the pump at $\Omega_{0}=$ $2 \omega_{\pi}$ thus makes magnon pairs around $k=\pi$ resonant, on a bandwidth of order $\sqrt{\mathcal{E} / \omega^{\prime \prime}} \pi_{\pi}$. For weak driving, where the bandwidth is low, higher-order terms in the dispersion relation can be neglected, and this simple calculation captures the amplification behaviour extremely well, as we illustrate in Fig. 2. We calculate the band structure and find the unstable modes numerically (see Methods), with $\tilde{\omega}_{k, s}$ and $\widetilde{\mathbf{Q}}_{s s^{\prime}}$ obtained from a microscopic model detailed in Supplementary Note 2, and plot the resulting band structure with instabilities in Fig. 1.

As we have seen above, an instability requires the anomalous terms to overcome the linear damping and the effective detuning. Linear damping, which we include as a uniform phenomenological parameter $\gamma$, has important consequences, as it sets a lower bound for the amplitude of the electrical field required to drive the system to an instability. It also ensures bulk stability. We have seen that there are three conditions for a parametric instability. First, there has to exist a pair of modes whose lattice momenta add to 0 (or $2 \pi$ ); Second, the sum of their energy has to match the pump frequency; and Third, the strength of their anomalous interaction has to overcome both their detuning and their damping. While momentum and energy matching is by design fulfilled by the edge mode, there is a large number of bulk mode pairs that also fulfil it. We show in Supplementary Note 4 that choosing the polarization to lie along $\gamma$ increases the anomalous coupling for modes with wavevector close to $\pi$ and that the coupling is small for almost all bulk mode pairs. The reason for this is that the bulk modes are approximately standing waves along $y$, and most bulk mode pairs have differing numbers of nodes, such that their overlap averages to zero. The remaining modes with appreciable anomalous coupling are far detuned in energy. This way, robust edge state instability can be achieved without any bulk instabilities, as demonstrated in Fig. 1. Bulk stability is crucial for the validity of the following discussion.

In the presence of an instability, the linear theory predicts exponential growth of edge magnon population. In a real system, the exponential growth is limited by nonlinear damping, for which we introduce another uniform parameter $\eta$, in the same spirit as Gilbert damping in nonlinear Landau-Lifshitz-Gilbert equations $^{21}$, such that Eq. (2) becomes

$$
i \frac{d}{d t} \mathbf{A}_{k}=\left(\begin{array}{cc}
\tilde{\omega}_{k}-i \frac{\gamma+\eta\left|\alpha_{k}\right|^{2}}{2} & \mathcal{E} \\
-\mathcal{E} & -\tilde{\omega}_{-k}-i \frac{\gamma+\eta\left|\alpha_{k}\right|^{2}}{2}
\end{array}\right) \mathbf{A}_{k} .
$$

Microscopically, such damping arises from the next order in the spin-wave expansion that allows four-wave mixing. While the linear theory only predicts the instability, Eq. (8) predicts a steady-state magnon occupation given through $\left|\alpha_{\pi+q}\right|^{2}=$ $\eta^{-1}\left(\sqrt{4 \mathcal{E}^{2}-q^{4}\left(\omega^{\prime \prime}\right)^{2}}-\gamma\right)$ (cf. Methods), which we show in Fig. 2b.

Experimental signatures. TMIs exhibit a magnonic thermal Hall effect at low temperatures ${ }^{4,5}$. A similar effect occurs when the magnon population is not thermal, but a consequence of coherent driving, realizing a driven Hall effect (DHE).

We can calculate the steady-state edge magnon current from the occupation calculated above,

$$
J_{\mathrm{SS}}=\int_{-\Lambda}^{\Lambda} \frac{d q}{2 \pi}\left|\alpha_{\pi+q}\right|^{2} \nu_{\pi+q},
$$

where $\nu_{\pi+q} \simeq \omega_{\pi}^{\prime}+q \omega_{\pi}^{\prime \prime}$ is the group velocity and $\Lambda=$ $\sqrt[4]{\left(4 \mathcal{E}^{2}-\gamma^{2}\right) /\left(\omega_{\pi}^{\prime \prime}\right)^{2}}$ is the range over which the steady-state population is finite (which coincides with the range over which the modes become unstable). While the integral can be done exactly ( $c f$. Methods) an approximation within $\pm 5 \%$ is given through

$$
J_{\text {SS }}(\mathcal{E}) \approx \frac{5 \omega_{\pi}^{\prime}}{6 \eta \pi}\left(\frac{4 \mathcal{E}^{2}-\gamma^{2}}{\left(\omega_{\pi}^{\prime \prime}\right)^{2}}\right)^{\frac{1}{4}}(2 \mathcal{E}-\gamma) .
$$

For $2 \mathcal{E} \gg \gamma$, a characteristic scaling of steady-state current with driving strength appears, $J_{\mathrm{SS}} \propto \mathcal{E}^{3 / 2}$, distinct from the linear dependence one would expect for standard heating.

In Fig. 3b, c, we demonstrate that the steady-state edge current depends on the drive strength in a fashion that is well described by Eq. (10). The order-of-magnitude equilibration time can be estimated from the solution to $\dot{\alpha}=(1 / 2)\left(\mathcal{E}-\eta|\alpha|^{2}\right) \alpha$, and for $\eta / \mathcal{E} \gg 1$ it evaluates to $t_{\mathrm{eq}} \sim \mathcal{E}^{-1} \log (\mathcal{E} / \eta) \sim 10^{4} J^{-1}$ for our chosen values of $\mathcal{E}$ and $\eta$. 
A DHE arises when a rectangular slab of size $L_{x} \times L_{y}$ is driven by a field with a gradient along $y$, as sketched in Fig. 3a. If $L_{x}$, $L_{y} \gg v_{\pi} t_{\text {eq }}$, the edges equilibrate to a steady-state magnon population governed by Eq. (10). The difference between the steady-state magnon currents on top and bottom edge corresponds to a net energy current $J_{\text {net }}^{x}$ along $x$, which to first order in the drive strength difference $\Delta_{y} \mathcal{E}$ can be written ${ }^{22}$

$$
J_{\text {net }}^{x}=\kappa_{x y}\left(\mathcal{E}_{\text {avg }}\right) \Delta_{y} \mathcal{E}, \quad \kappa_{x y}(\mathcal{E}) \equiv \frac{d J_{\text {SS }}(\mathcal{E})}{d \mathcal{E}}
$$

where one should note that in this non-equilibrium setting $\kappa_{x y}$ is not a proper conductivity as in conventional linear response. The net edge current causes one side of the system to heat up faster, resulting in a temperature difference transverse to the gradient. As the edge magnons decay along the edge, the reverse heat current is carried by bulk modes. For small temperature differences the heat current follows the temperature gradient linearly and thus the averaged temperature difference $\Delta_{x} T=\int d y\left[T\left(L_{x}, y\right)-T(0, y)\right] / L_{y}=J_{\text {net }}^{x} / \kappa_{x x}$. The temperature difference can thus be written in terms of the applied field strength difference

$$
\Delta_{x} T=\frac{\Delta_{y} \mathcal{E}}{\kappa_{x x}} \kappa_{x y}(\mathcal{E})
$$

As a word of caution, we note that this relation relies on several key assumptions. To begin with, temperature is in fact not well defined along the edge, as there is a non-equilibrium magnon occupation. Edge magnons decay at a certain rate into phonons, which can be modelled as heating of the phonon bath. If the equilibration time scale of the latter is fast compared to the heating rate through magnon decay, one can at least associate a local temperature to the phonons. Similarly, the bulk magnon modes can be viewed as a fast bath for the magnon edge mode and similar considerations apply. Even if these assumptions are justified, the two baths do not need to have the same temperature. Next, the heat conductivity associated to magnons and phonons differ in general, such that the $\kappa_{x x}$ appearing in Eq. (12) can only be associated with the bulk heat conductivity if the temperatures of the two baths are equal. Some of these complications have been recognized to also play an important role in measurements of the magnon thermal Hall effect ${ }^{22}$.

While the above-mentioned concerns make quantitative predictions difficult, the DHE is easily distinguishable from the thermal Hall effect, due to the strong dependence of the temperature difference $\Delta_{x} T$ on drive frequency and polarization, as well as the fact that below the cutoff $2 \mathcal{E}=\gamma$ no instability occurs and that $J_{\text {net }} \propto \sqrt{\mathcal{E}_{\text {avg }}}$ for $2 \mathcal{E}_{\text {avg }} \gg \gamma$, rather than the linear dependence one would expect from standard heating. In certain materials such as $\mathrm{Cu}(1,3-\mathrm{bdc})$, the appearance or disappearance of the topological edge modes can be tuned with an applied magnetic field ${ }^{12}$, a property that could be used to further corroborate the results of such an experiment.

A number of other experimental probes might be used to certify a large edge magnon current and thus the presence of edge states. On the one hand, with a large coherent magnon population in a given mode, the local magnetic field and electric polarization associated to that mode will be enhanced. In particular, techniques that directly probe local magnetic or electric fields, such as neutron scattering ${ }^{12,23}$ or $\mathrm{x}$-ray scattering, which to date are not powerful enough to resolve edge modes ${ }^{12}$, would thus have a coherently enhanced signal, for example, by almost two orders of magnitude when taking the conservative parameters in Fig. 2. On the other hand, heterostructures provide a way to couple the magnons out of the edge mode into another material ${ }^{21}$, for example one with a strong spin Hall effect, in which they can be detected more easily. In this setup, again the fact that the edge magnons have a large coherent population should make their signal easily distinguishable from thermal noise.

Material realizations. The model of a kagome lattice ferromagnet with DM interaction has been found to describe the thermal magnon Hall effect in $\mathrm{Lu}_{2} \mathrm{~V}_{2} \mathrm{O}_{7}{ }^{5}$, as well as the bulk magnon band structure of $\mathrm{Cu}(1,3-\mathrm{bdc})^{12,24}$. These materials are in fact $3 \mathrm{D}$ pyrochlore lattices, which can be pictured as alternating kagome and triangular lattices along the [111] direction. However, their topological properties can be captured by considering only the kagome planes $^{10-12}$ (shown in Fig. 1), thus neglecting the coupling between kagome and triangular planes. It has been suggested that the effect of the interaction may be subsumed into new effective interaction strengths ${ }^{11}$ or into an effective on-site potential ${ }^{10}$. Typical values for strength of the DM and Heisenberg interactions lie between $|\mathbf{D}| / J \approx 0.18^{12}, J \approx 0.6 \pm 0.1 \mathrm{meV} \simeq 150 \pm 30 \mathrm{GHz}^{12}$ in $\mathrm{Cu}(1,3-\mathrm{bdc})$ and $|\mathbf{D}| / J \approx 0.32^{5}, J \approx 3.4 \mathrm{meV} \approx 0.82 \mathrm{THz}^{10}$ in $\mathrm{Lu}_{2} \mathrm{~V}_{2} \mathrm{O}_{7}$. The energy of the edge states close to $k=\pi$ is approximately $J$, such that the applied drive needs to be at a frequency $\omega_{0} / 2 \pi=0.3-1.6 \mathrm{THz}$. While experimentally challenging, low $\mathrm{THz}$ driving down to 0.6 $\mathrm{THz}$ has recently been achieved ${ }^{18,25}$. Furthermore, the magnon energy can be tuned by applied magnetic fields.

An instability requires $E_{0} a e(t / U)^{3} \gtrsim \gamma$. With $a \simeq 10 \AA^{10}, J \simeq 1$ $\mathrm{meV}$, and assuming $t / U \simeq 0.1$, we can estimate the minimum field strength required to overcome damping $\gamma_{k} \simeq 10^{-4} \mathrm{~J}$ to be $E_{0} \simeq 10^{5}$ $\mathrm{V} / \mathrm{m}$, although for quantitative estimates one would require both accurate values for the damping of the edge modes (at zero temperature) and $t / U$. This is accessible in pulsed operation ${ }^{18,25}$, and perhaps in continuous operation through the assistance of a cavity.

Since the qualitative behaviour we describe can be derived from general and phenomenological considerations, we expect it to be robust and present in a range of systems, as long as they allow for anisotropy, i.e., if bonds are not centres of inversion. We thus expect that topological magnon amplification is also possible in recently discovered topological honeycomb ferromagnet $\mathrm{CrI}_{3}{ }^{26}$.

\section{Discussion}

We have shown that appropriate electromagnetic driving can render topological magnon edge modes unstable, while leaving the bulk modes stable. The resulting non-equilibrium steady state has a macroscopic edge magnon population. We present several strategies to certify the topological nature of the band structure, namely, implementing a driven Hall effect (DHE), direct detection with neutron scattering, or by coupling the magnons into a material with a spin Hall effect.

Our work paves the way for a number of future studies. As we have pointed out, edge mode damping plays an important role here. One might expect their damping to be smaller than that of generic bulk modes as due to their localization they have a smaller overlap to bulk modes. This suppression should be compounded by the effect of disorder ${ }^{21}$, which may further enhance the feasibility of our proposed experiments. On the other hand, rough edges will have an influence over the matrix element between drive and edge modes, leading to variations in the anomalous coupling strength. Phonons in the material are crucial for robust thermal Hall measurements ${ }^{22}$ and could possibly mix with the chiral magnon mode ${ }^{27}$, which motivates full microscopic calculations.

An exciting prospect is to use topological magnon amplification in magnon spintronics. There have already been theoretical efforts studying how magnons can be injected into topological edge modes with the inverse spin Hall effect ${ }^{21}$. Given an efficient 
mechanism to couple magnons into and out of the edge modes, our amplification mechanism may enable chiral travelling-wave magnon amplifiers, initially proposed in photonic crystals ${ }^{9}$. Even when simply seeded by thermal or quantum fluctuations, the large coherent magnon steady state could power topological magnon lasers ${ }^{14}$, with tremendous promise for future application in spintronics. In the near future, we hope that topological magnon amplification can be used for an unambiguous discovery of topological magnon edge modes.

\section{Methods}

Numerical calculation. For the numerical calculation, we choose a manifestly inversion-symmetric system obtained by deleting the lowest row of sites, a situation that is depicted in Fig. 1, where the tip of the lowest blue triangle is part of a unit cell whose other sites are not included. For example, repeating the star shown in Fig. 1 along $x$ would result in an inversion-symmetric strip with $W=3$. A Fourier transform of Eq. (5) along $x$ yields a $3 W-2$ by $3 W-2$ Hamiltonian matrix for each momentum $k$

$$
\begin{aligned}
H_{0}= & K_{0}-\frac{1}{2}\left\{( J + i D _ { z } ) \sum _ { k , l _ { y } } \left[e^{-i k / 4} a_{1, k, l_{y}}^{\dagger} a_{2, k, l_{y}}\right.\right. \\
& +e^{-i k / 4} a_{2, k, l_{y}}^{\dagger} a_{3, k, l_{y}}+2 \cos (k / 2) a_{3, k, l_{y}}^{\dagger} a_{1, k, l_{y}} \\
+ & \left.\left.e^{i k / 4} a_{1, k, l_{y}}^{\dagger} a_{2, k, l_{y}-1}+e^{i k / 4} a_{2, k, l_{y}}^{\dagger} a_{3, k, l_{y}+1}\right]+ \text { H.c. }\right\}
\end{aligned}
$$

Note that we take $\hbar=1$. Diagonalizing this matrix yields single-particle energy eigenstates with annihilation operator $b_{k, s}$ and a Hamiltonian $H_{0}=\sum_{k, s} \omega_{k, s} b_{k, s}^{\dagger} b_{k, s}$.

The resulting band structure is shown in Fig. 1. In our convention, the lowest bulk band has Chern number $\operatorname{sgn} D_{z}$, the middle bulk band 0 and the top bulk band $-\operatorname{sgn} D_{z}$ (calculated, e.g., through the method described in ref. ${ }^{28}$ ). Accordingly, there is one pair of edge modes in each of the bulk gaps, one right-moving localized at the lower edge and one left-moving at the upper.

Including the anomalous terms obtained from a calculation based on the FermiHubbard model at half filling yields the full Hamiltonian Eq. (7). By means of a Bogoliubov transformation we obtain the magnon band structure and the unstable states $^{29}$, which form the basis for Fig. 2. The inclusion of nonlinear damping yields Eq. (8), which has been used to calculate Fig. 3. In the end, we calculate the current by evaluating the expectation value of the particle current or energy current operator, which can be obtained for a given bond from the continuity equation ${ }^{30}$. The current across a certain cut of the system is obtained by summing the current operators for all the bonds that cross it. As the system we study is inversion symmetric, the total current in the $x$ direction vanishes. In order to specifically find the edge current, we thus define a cut through half of the system, for example from the top edge to the middle.

Unstable modes in Bogoliubov-de Gennes equation. We consider the full Hamiltonian

$$
H=H_{0}-H_{\text {rot }}+H_{\text {amp }} .
$$

$H_{0}-H_{\text {rot }}$ gives rise to the band structure shown in Fig. 1 above, while $H_{\text {amp }}$ contains the anomalous terms. The idea of this section is to calculate which modes in Eq. (14) are unstable. Ideally, those should be the relevant edge modes, and only those. It turns out that this is possible in presence of linear damping.

Following ref. ${ }^{9}$, we define the vector

$a_{k}=\left(a_{k, 1}, a_{k, 2}, \ldots, a_{k, N}, a_{k_{0}-k, 1}^{\dagger}, \ldots, a_{k_{0}-k, N}^{\dagger}\right)^{T}$, where the index combines the label $l_{y}$ and the site label in the unit cell and therefore runs from 1 to $N=3 W-2$. The Hamiltonian can generically be written

$$
H=\sum_{k}\left[a_{k, s}^{\dagger} \mu_{k, s s^{\prime}} a_{k, s^{\prime}}+\frac{1}{2}\left(a_{k, s}^{\dagger} \nu_{k, s s^{\prime}} a_{-k, s^{\prime}}^{\dagger}+\text { H.c. }\right)\right]
$$

where $\mu_{k}$ originates from $H_{0}-H_{\text {rot }}$ and $v_{k}$ from $H_{\text {amp. }}$. This form makes it evident that $\mu_{k}=\mu_{k}^{\dagger}$ and $\nu_{k}=\nu_{k}^{T}$. The equation of motion for this vector can be found from the Hamiltonian above and is

$$
\frac{d}{d t}\left|a_{k}\right\rangle=-i \sigma_{z} h_{k}\left|a_{k}\right\rangle
$$

with $\sigma_{z}=\operatorname{diag}(1,1, \ldots,-1,-1, \ldots)(N$ “ +1 ”s and $N$ “ -1 ”s), with

$$
h_{k}=\left(\begin{array}{cc}
\mu_{k} & \nu_{k} \\
\nu_{k}^{\dagger} & \mu_{-k}^{T}
\end{array}\right) .
$$

We can then solve the eigenvalue problem and find stable and unstable modes. Furthermore, we can find the time-evolution for operators in the Heisenberg picture from Eq. (16). It is simply $\left|a_{k}(t)\right\rangle=e^{-i \sigma_{z} h_{k} t}\left|a_{k}(0)\right\rangle$.
Steady state of nonlinear equations of motion. We start from the equations of motion (8) given in the main text, repeated here for convenience

$$
i\left(\begin{array}{c}
\dot{\alpha}_{k} \\
\dot{\alpha}_{-k}^{*}
\end{array}\right)=\left(\begin{array}{cc}
\tilde{\omega}_{k}-i \frac{\gamma+\eta\left|\alpha_{k}\right|^{2}}{2} & \mathcal{E} \\
-\mathcal{E} & -\tilde{\omega}_{-k}-i \frac{\gamma+\eta\left|\alpha_{k}\right|^{2}}{2}
\end{array}\right)\left(\begin{array}{c}
\alpha_{k} \\
\alpha_{-k}^{*}
\end{array}\right)
$$

In the steady state, $\left|\alpha_{k}\right|^{2}=$ const., so we use the ansatz $\alpha_{k}=\exp (i \Delta t) \bar{\alpha}$, and $\alpha_{-k}^{*}=\exp (i \Delta t) z \bar{\alpha}$ for some complex numbers $z, \bar{\alpha}$ and real frequency $\Delta$. As we are only interested in a narrow range of momenta, we expand the dispersion relation to second order, as in the main text. The pump frequency is set to match the edge mode at $k=\pi$, i.e., $\Omega_{0}=2 \omega_{\pi}$. As a consequence, $\tilde{\omega}_{\pi+q}=q \omega_{\pi}^{\prime}+q^{2} \omega_{\pi}^{\prime \prime} / 2+\mathcal{O}\left(q^{3}\right)$.

If there is an instability, the solution $\bar{\alpha}=0$ is unstable. Assuming $\bar{\alpha} \neq 0$ (thus $z \neq 0)$, and for $\Delta=q \omega_{\pi}^{\prime}$, we find the set of equations

$$
\begin{aligned}
& \frac{1}{2} q^{2} \omega_{\pi}^{\prime \prime}-\frac{i}{2}\left(\gamma+\eta\left|\alpha_{k}\right|^{2}\right)+z \mathcal{E}=0, \\
& -\frac{1}{2} q^{2} \omega_{\pi}^{\prime \prime}-\frac{i}{2}\left(\gamma+\eta\left|z \alpha_{k}\right|^{2}\right)-\frac{\mathcal{E}}{z}=0 .
\end{aligned}
$$

Multiplying the second equation by $|z|^{2}$, and subtracting the complex conjugate of the resulting equation from the first equation, one can show that $|z|^{2}=1$. With this condition Eqs. (19) and (20) coincide, such that we can solve them for the intensity

$$
\left|\alpha_{k}\right|^{2}=\frac{1}{\eta}\left(-2 i \mathcal{E} z-i q^{2} \omega_{\pi}^{\prime \prime}-\gamma\right) \text {. }
$$

This equation has solutions if and only if $4 \mathcal{E}^{2} \geq q^{4}\left(\omega_{\pi}^{\prime \prime}\right)^{2}+\gamma^{2}$, which coincides with the condition for the instability. If this condition is fulfilled, we have

$$
\left|\alpha_{\pi+q}\right|^{2}=\frac{1}{\eta}\left(\sqrt{4 \mathcal{E}^{2}-q^{4}\left(\omega_{\pi}^{\prime \prime}\right)^{2}}-\gamma\right) .
$$

The steady-state edge magnon current

$$
\begin{aligned}
J_{\text {SS }} & =\int_{-\Lambda}^{\Lambda} \frac{d q}{2 \pi}\left|\alpha_{\pi+q}\right|^{2} \nu_{\pi+q} \\
& =\frac{2 \omega_{\pi}^{\prime} \sqrt{2 \mathcal{E}}}{3 \pi \eta \sqrt{\omega_{\pi}^{\prime \prime}}}\left\{2 \mathcal{E} F\left[\sin ^{-1}\left(\frac{\Lambda}{\sqrt{2 \mathcal{E} / \omega_{\pi}^{\prime \prime}}}\right),-1\right] \gamma \sqrt[4]{1-\gamma^{2} /\left(4 \mathcal{E}^{2}\right)}\right\}
\end{aligned}
$$

where $F(k, m)$ is the elliptic integral of the first kind.

Particle current operator. The particle current operator is obtained from the continuity equation for the number of magnons. We have

$$
\dot{n}_{n}-i\left[H_{0}, n_{n}\right]=\dot{n}_{n}-i \sum_{m}\left[h_{m}, n_{n}\right]=0,
$$

where $h_{n}$ are local Hamiltonians defined through

$$
H_{0}=\sum_{n} h_{n}
$$

The second term in Eq. (24) can be interpreted as a sum of the particle currents from $n$ to the neighbouring sites $m$.

\section{Data availability}

Data sharing not applicable to this article as no datasets were generated or analysed during the current study.

Received: 9 May 2019 Accepted: 5 August 2019

Published online: 02 September 2019

\section{References}

1. Hasan, M. Z. \& Kane, C. L. Colloquium: topological insulators. Rev. Mod. Phys. 82, 3045-3067 (2010).

2. Qi, X.-L. \& Zhang, S.-C. Topological insulators and superconductors. Rev. Mod. Phys. 83, 1057-1110 (2011).

3. Vishwanath, A. \& Senthil, T. Physics of three-dimensional bosonic topological insulators: surface-deconfined criticality and quantized magnetoelectric effect. Phys. Rev. X 3, 011016 (2013).

4. Katsura, H., Nagaosa, N. \& Lee, P. A. Theory of the thermal Hall effect in quantum magnets. Phys. Rev. Lett. 104, 066403 (2010).

5. Onose, Y. et al. Observation of the magnon Hall effect. Science 329, 297-299 (2010).

6. Hirschberger, M., Chisnell, R., Lee, Y. S. \& Ong, N. P. Thermal Hall effect of spin excitations in a kagome magnet. Phys. Rev. Lett. 115, 106603 (2015).

7. Haldane, F. D. M. \& Raghu, S. Possible realization of directional optical waveguides in photonic crystals with broken time-reversal symmetry. Phys. Rev. Lett. 100, 013904 (2008). 
8. Wang, Z., Chong, Y., Joannopoulos, J. D. \& Soljačić, M. Observation of unidirectional backscattering-immune topological electromagnetic states. Nature 461, 772-775 (2009).

9. Peano, V., Houde, M., Marquardt, F. \& Clerk, A. A. Topological quantum fluctuations and traveling wave amplifiers. Phys. Rev. X 6, 041026 (2016).

10. Zhang, L., Ren, J., Wang, J.-S. \& Li, B. Topological magnon insulator in insulating ferromagnet. Phys. Rev. B 87, 144101 (2013).

11. Mook, A., Henk, J. \& Mertig, I. Magnon Hall effect and topology in kagome lattices: a theoretical investigation. Phys. Rev. B 89, 134409 (2014).

12. Chisnell, R. et al. Topological magnon bands in a kagome lattice ferromagnet. Phys. Rev. Lett. 115, 147201 (2015).

13. Chumak, A. V., Vasyuchka, V. I., Serga, A. A. \& Hillebrands, B. Magnon spintronics. Nat. Phys. 11, 453-461 (2015).

14. Harari, G. et al. Topological insulator laser: Theory. Science 359, eaar4003 (2018).

15. Bandres, M. A. et al. Topological insulator laser: experiments. Science 359, eaar4005 (2018).

16. Galilo, B., Lee, D. K. \& Barnett, R. Selective population of edge states in a 2D topological band system. Phys. Rev. Lett. 115, 245302 (2015).

17. Galilo, B., Lee, D. K. \& Barnett, R. Topological edge-state manifestation of interacting $2 \mathrm{D}$ condensed boson-lattice systems in a harmonic trap. Phys. Rev. Lett. 119, 203204 (2017).

18. H. Plank, et al. Edge currents driven by terahertz radiation in graphene in quantum Hall regime, Preprint at http://arxiv.org/abs/1807.01525 (2018).

19. Chernyshev, A. L. \& Maksimov, P. A. Damped topological magnons in the kagome-lattice ferromagnets. Phys. Rev. Lett. 117, 187203 (2016).

20. Moriya, T. Theory of absorption and scattering of light by magnetic crystals. J. Appl. Phys. 39, 1042-1049 (1968).

21. Rückriegel, A., Brataas, A. \& Duine, R. A. Bulk and edge spin transport in topological magnon insulators. Phys. Rev. B 97, 081106 (2018).

22. Vinkler-Aviv, Y. \& Rosch, A. Approximately quantized thermal Hall effect of chiral liquids coupled to phonons. Phys. Rev. X 8, 031032 (2018).

23. Yao, W. et al. Topological spin excitations in a three-dimensional antiferromagnet. Nat. Phys. 14, 1011-1015 (2018).

24. Nytko, E. A., Helton, J. S., Müller, P. \& Nocera, D. G. A structurally perfect $S$ $=1 / 2$ Metal-Organic Hybrid Kagomé Antiferromagnet. J. Am. Chem. Soc. 130, 2922-2923 (2008).

25. Karch, J. et al. Terahertz radiation driven chiral edge currents in graphene. Phys. Rev. Lett. 107, 276601 (2011).

26. Chen, L. et al. Topological spin excitations in honeycomb ferromagnet $\mathrm{CrI}_{3}$. Phys. Rev. X 8, 041028 (2018).

27. Thingstad, E., Kamra, A., Brataas, A. \& Sudbø, A. Chiral phonon transport induced by topological magnons. Phys. Rev. Lett. 122, 107201 (2019).

28. Fukui, T., Hatsugai, Y. \& Suzuki, H. Chern numbers in discretized brillouin zone: efficient method of computing (Spin) Hall conductances. J. Phys. Soc. Jpn. 74, 1674-1677 (2005).

29. Blaizot, J. P. \& Ripka, G. Quantum Theory of Finite Systems. (The MIT Press, Cambridge, MA, 1986).
30. Bernevig, B. A. \& Hughes, T. L. Topological Insulators and Topological Superconductors, edition. 1sted (Princeton University Press, Princeton, New Jersey, 2013).

\section{Acknowledgements}

We are grateful to Ryan Barnett, Derek Lee, Rubén Otxoa, Pierre Roy, and Koji Usami for insightful discussions and helpful comments. D.M. acknowledges support by the Horizon 2020 ERC Advanced Grant QUENOCOBA (grant agreement 742102). AN holds a University Research Fellowship from the Royal Society and acknowledges support from the Winton Programme for the Physics of Sustainability and the European Union's Horizon 2020 research and innovation programme under grant agreement No 732894 (FET Proactive HOT).

\section{Author contributions}

D.M. performed the analysis and wrote the manuscript with assistance from J.K. and A.N. All authors contributed to the conception.

\section{Additional information}

Supplementary Information accompanies this paper at https://doi.org/10.1038/s41467 019-11914-2.

Competing interests: The authors declare no competing interests.

Reprints and permission information is available online at http://npg.nature.com/ reprintsandpermissions/

Peer review information: Nature Communications thanks Tomoki Ozawa and other anonymous reviewer(s) for their contribution to the peer review of this work.

Publisher's note: Springer Nature remains neutral with regard to jurisdictional claims in published maps and institutional affiliations.

Open Access This article is licensed under a Creative Commons Attribution 4.0 International License, which permits use, sharing, adaptation, distribution and reproduction in any medium or format, as long as you give appropriate credit to the original author(s) and the source, provide a link to the Creative Commons license, and indicate if changes were made. The images or other third party material in this article are included in the article's Creative Commons license, unless indicated otherwise in a credit line to the material. If material is not included in the article's Creative Commons license and your intended use is not permitted by statutory regulation or exceeds the permitted use, you will need to obtain permission directly from the copyright holder. To view a copy of this license, visit http://creativecommons.org/ licenses/by/4.0/.

(c) The Author(s) 2019 